(Physiologisches Laboratorium in Bonn.)

\title{
Fortgesetzte Untersuchung über den Glykogengehalt der foetalen Leber und die Jodreaction des Glykogenes.
}

Von

\section{E. Pfliiger.}

\section{$\$ 1$. Ueber den Glykogengehalt der foetalen Leber.}

Bekanntlich gelangte Claude Bernard ${ }^{1}$ ) durch die Untersuchung der verschiedenen Organe der Embryonen zu der Ansicht, dass die Leber in der ersten Hälfte des foetalen Lebens kein Glykogen enthalte, obwohl die Muskeln, Lungen u. s. w. erhebliche Mengen beherbergen. Weil diese Behauptung so räthselhaft ist, entschloss ich mich, sie genauer zu prüfen.

Claude Bernard entzog der Leber das Glykogen mit siedendem Wasser. Heute weiss man, dass man auf diese Weise recht erhebliche Mengen von Glykngen übersehen kann, weshalb ich die Frage in der Art prüfte, dass ich die foetale Leber mit siedender Kalilauge löste und daraus das Glykogen mit Alkohol. fällte.

Ich berichtete bereits in Bd. 95 S. 19 dieses Archives über meine ersten Ergebnisse, welche bewiesen, dass in der Leber von Embryonen, die sich ungefähr in dem zweiten Viertel des foetalen Lebens befinden, das Glykogen niemals vermisst wurde. Wenn nun auch zuweilen in der foetalen Leber nicht unerhebliche Mengen (bis zu $0,79 \%$ ) Glykogen nachgewiesen werden konnten, war es doch auffallend, dass in der Regel der Gehalt unter $0,1 \%$ lag, ja bis auf Spuren herabsank, während in den Muskeln beträchtliche Mengen von Glykogen niemals fehlten.

Zur richtigen Beurtheilung dieser auf den ersten Blick auffallenden Thatsachen ist aber zu beachten, dass die Leber der Kälber

1) Claude Bernard, Journal de la Physiologie t. 2 p. 385.1859. 
nach der Geburt - wenigstens in Bonn - gewöhnlich ebenfalls sehr arm an Glykogen gefunden wird, obwohl die Muskeln daran reich sein können. Ich zeigte durch Fütterungsversuche, dass auch die Leber dieser Kälber reich an Glykogen wird, wenn man die Thiere einige Tage vor dem Schlachten gut ernährt. Die Armuth der Leber an Glykogen ist bei diesen Thieren also sicher nur durch mangelhafte Ernährung bedingt. Aehnlich verhält es sich mit dem Pferde. Die Leber erweist sich der Regel nach als sehr arm, die Muskeln sehr reich an Glykogen. Auch hier habe ich durch besondere Fütterungsversuche festgestellt, dass die Leber der Pferde bei guter Ernährung sehr reich an Glykogen wird.

Weil man bei Analysirung der foetalen Leber aus den frühen Entwicklungsperioden, um ausreichendes Material zu baben, auf grosse Thiere - also die Kuh - angewiesen ist, und nur der $\mathrm{Zu}$ fall die Embryonen von Thieren zuführt, deren Trächtigkeit vor dem Schlachten nicht bekannt war, so fehlte mir die Möglichkeit, die trächtige Kuh vor der Tödtung einige Tage reichlich mit Kohlehydraten zu füttern. Bei solcher Fütterung würde es sich zeigen, ob die Leber der jungen Embryonen im Stande ist, so grosse procentige Glykogenmengen aufzuhäufen, wie die der Thiere nach der Geburt.

Möglich bleibt also vor der Hand nur die Untersuchung, ob grundsätzlich, wie Claude-Bernard behauptet hat, die foetale Leber in den frühen Entwicklungsperioden überhaupt gar kein Glykogen enthält.

Ich hatte bis jetzt noch nicht die Lebern aus dem ersten Viertel der Embryonalzeit untersucht, und ich setzte mir deshalb vor, bis zu den frühesten Perioden zurückzugreifen, in denen die Kleinheit der Leber noch nicht dem chemischen Nachweise eine natürliche Grenze setzt.

Ich gebe in der Tabelle auf S. 307 eine Zusammenstellung der Ergebnisse an den Foeten, welche ich untersuchen konnte.

In sämmtlichen Lebern liess sich Glykogen sicher nachweisen, sowohl durch die Jodreaction als durch die Bildung von Zucker nach Inversion der Glykogenlösung mit Salzsäure von 2,2\%. Beim Kalbfoetus von $13 \mathrm{ccm}$ resp. 5 Wochen Alter liess sich aus der $2,6 \mathrm{~g}$ schweren Leber ein Extract gewinnen, welches die Jodreaction nicht mit Sicherheit festzusetzen erlaubte. - Dahingegen lieferte die Leber des $25 \mathrm{~cm}$ langen Kalbsfoetus, der angeblich 6 Wochen alt 
Fortges. Untersuchung über den Glykogengehalt der foetalen Leber etc. 307

war, ein Extract, welches sofort mit Jod tief rothbraun wurde; beim Erhitzen verschwand die Farbe, kehrte aber beim Abkühlen kräftig zurück. - Dasselbe Extract lieferte nach Inversion bei Ausführung der Zuckerreaction in später beschriebener Weise eine beträchtliche Abscheidung von Kupferoxydul.

\begin{tabular}{|c|c|c|c|c|}
\hline $\begin{array}{l}\text { Art des } \\
\text { Thieres }\end{array}$ & $\begin{array}{c}\text { Länge des } \\
\text { Thieres } \\
\text { (von Schnauze } \\
\text { bis Schwanz- } \\
\text { wurzel) }\end{array}$ & $\begin{array}{c}\text { Angebliches } \\
\text { Alter des } \\
\text { Thieres }\end{array}$ & $\begin{array}{c}\text { Gewicht } \\
\text { der } \\
\text { Leber }\end{array}$ & $\begin{array}{c}\text { Normale } \\
\text { Dauer der } \\
\text { Trächtigkeit }\end{array}$ \\
\hline Kalbsfoetus & $13 \mathrm{ccm}$ & 5 Wochen & $2.6 \mathrm{~g}$ & 9 Monate \\
\hline Kalbsfoetus & 25 & 6 Wochen & 23,0 & 9 Monate \\
\hline Kalbsfoetus & $31 "$ & 8 Wochen & 51,0 & 9 Monate \\
\hline Kalbsfoetus & 31 & 7 Wochen & $51,0 "$ & 9 Monate \\
\hline Kalbsfoetus & 34 & 11 Wochen & $84,0 "$ & 9 Monate \\
\hline
\end{tabular}

Die wichtigste Thatsachenrejhe besteht darin, dass die embryonale Leber ganz ausserordentlich grosse Schwankungen im Glykogengehalte darbietet, so dass bald reichliche Mengen, bald nur Spuren gefunden werden, während die Muskeln stets beträchtliche Vorräthe an Glykogen darbieten. Es ist genau dasselbe Verhältniss, wie ich es bei dem erwachsenen Pferde gefunden habe, und hier liegt die Ursache unzweifelhaft in dem Ernährungszustande des Thieres. Die Leber ist eine grosse Vorrathskammer, welche bei guter Nahrungszufuhr Massen von Glykogen aufspeichert, welche zur Zeit des Mangels mit grösster Freigebigkeit an die verschiedenen Organe des Körpers abgegeben werden. Es ist etwas ganz Gewöhnliches, dass die Muskeln der geschlachteten Pferde 2\% Glykogen, die Leber aber nur einige Zehntel Procent enthält. Es liegt deshalb nahe, anzunehmen, dass es sich beim Einbryo genau ebenso verhält, weil auch seine Leber die uneigennützige Vorrathskammer zur Ernährung des übrigen Körpers darstellt.

Der Nachweis des Glykogens der foetalen Leber macht grössere Schwierigkeiten, weshalb ich folgende Vorschrift empfehle, die ich zur Erleichterung des Verständnisses auf $100 \mathrm{~g}$ Leber beziehe.

$100 \mathrm{~g}$ Leber werden mit $100 \mathrm{ccm}$ Kalilauge von $60 \% \mathrm{im}$ Wasserbad erhitzt und dann abgekühlt. Die auf $200 \mathrm{ccm}$ aufgefüllte Organlösung versetzte ich mit $200 \mathrm{ccm}$ Wasser und diese $400 \mathrm{cem}$ Mischung mit $400 \mathrm{cem}$ Alkohol von $96 \%$ Tr. Man ver- 
fährt also im Wesentlichen so, wie ich es in meiner Abhandlung ${ }^{1}$ ) über die quantitative Glykogenanalyse vorgeschlagen habe. Besonders zu beachten ist aber, dass das Kochen der foetalen Leber mit Kalilauge eine Reihe von Stunden fortgesetzt werden soll, um die Eiweissstoffe ihrer Fällbarkeit durch Alkohol möglichst zu berauben, was trotzdem nicht vollständig gelingt. - Es ist ferner zu beachten, dass die nach der Verdünnung mit Wasser erhaltene trübe Lösung nicht filtrirt wird, ehe man den Alkohol zur Fällung hinzufügt. Warum dies vorgeschrieben wird, kann erst in einer später zu veröffentlichenden Abhandlung gereehtfertigt werden.

Nach einer Reihe von Stunden setzt sich ein braunes Sediment $a b$, welches (am Besten nach ungefähr 24 Stunden) durch ein schwedisches Filter abfiltrirt wird. Sobald die Flüssigkeit beinahe abgetropft ist, wäscht man das Glykogen mit einer Lösung, welche besteht aus

$$
\begin{aligned}
& 1 \text { Vol. Lauge von } 15 \% \mathrm{KOH} \\
& 2 \% \text { Alkohol , } 96 \% \mathrm{Tr} \text {. }
\end{aligned}
$$

Man bringt diese Waschlösung in das Becherglas, in dem die Glykogenfällung vollzogen worden ist, und giesst daraus zwei Mal auf das Filter. - Nach Abtropfen giesst man Alkohol von $96 \%$ auf das Filter, wodurch das Glykogen zum Schrumpfen gebracht wird. Nach Abtropfen des Alkohols giesst man sterilisirtes Wasser auf und fährt so lange damit fort, als das Filtrat sich noch mit Alkohol trübt. Diese Filtration nimmt eine Reihe von Stunden in Anspruch, weil schleimige Flocken die Poren des Filters mehr oder weniger verstopfen.

\section{$\S 2$. Ueber die Vorbereitung des Leberauszuges für die Jod- reaction des Glykogenes.}

Es handelt sich jetzt darum, zuerst die Jodreaction anf Glykogen anzustellen. Ich habe in letzter Zeit gefunden, dass die Gegenwart von Säuren und ebenso von Alkohol die Schärfe dieser Reaction beeinträchtigt.

Demgemäss dampfe ich das alkalische Filtrat auf dem Wasserbad in einer Glasschale auf ein kleines Volum von 20 bis $50 \mathrm{ccm}$ ein, um den Alkohol zu verjagen, lasse abkühlen und neutralisire mit sehr verdünnter Essigsäure höchst sorgfältig, füge endlich noch

1) E. Pflüger, dieses Archiv Bd. 93 S. 163. 
Fortges. Untersuchung über den Glykogengehalt der foetalen Leber etc. 309 ein wenig Essigsäure zu, bis ich die erste Spur saurer Reaction erhalte.

Es haben sich Eiweissflöckchen ausgeschieden, welche durch ein sehr kleines nasses Filter abfiltrirt werden. Das klare Filtrat dient nun zuerst zur Anstellung der Jodreaction.

Ehe ich zur Beschreibung der hier in Betracht kommenden Erscheinungen übergehe, will ich eine kleine Untersuchung mittheilen, die ich über die Jodglykogenreaction angestellt habe.

\section{\$ 3. Die Jodreaction mit reinem Glykogen.}

Zuerst sei daran erinnert, dass es sich bei dieser um eine Reihe sehr verschiedener Farben handelt, deren Ton von der Concentration der Jodglykogenlösung abhängt, beginnend mit blassem Gelbbraun, das durch Rothbraun in das schönste tiefste Roth übergeht. In rünnster Schicht hat aber diese concentrirte Lösung einen Stich in's Braune, besonders deutlich, wenn es sich um das Glykogen der Leber handelt.

Weil die Jodlösung auch ohne Gegenwart von Glykogen einer farblosen Flüssigkeit einen gelbröthlichen Farbenton ertheilt, muss die Jod-Glykogenreaction stets controlirt werden wie ich schon früher jmmer hervorhob ${ }^{1}$ ).

Man füllt zwei Reagensgläser gleichen Calibers mit gleichen Mengen derselben Jodlösung und fügt dann einen Tropfen Glykogenlösung einem der Reagensgläser zu.

Noch zweckmässiger ist es, wie Madame Gatin-Gružewska in meinem Laboratorium gefunden und hier mitzutheilen erlaubt hat, wenn man zwei Reagensgläser gleichen Calibers so beschickt, dass man in das eine Reagensglas Wasser und in das zweite ein gleiches Volum der auf Glykogen zu untersuchenden Lösung bringt und dann in jedes Reagensglas aus einer Bürette je einen Tropfen einer sehr concentrirten Jodlösung fallen lässt.

Erhitzt man nun beide Reagensgläser gleichzeitig: gleichstark und gleichlang, so ergibt sich die wichtige Thatsache, dass der noch so grosse anfänglich vorhandene Farbenunterschied vollkommen verschwindet. Beim Kochen einer Jodglykogenlösung tritt also zuletzt die Farbe

1) E. Pflüger, dieses Archiv Bd. 75 S. 198.1899. 
auf, welche die Lösung schon hatte, ehe das Glykogen hinzugesetzt wurde. Diese Farve ist folglich nur durch das freigewordene Jod bedingt; dies zeigt, dass die Jodglykogenverbindung sich beim Erhitzen vollkommen zersetzt. Da beim Abkühlen die Farbe der Jodglykogenverbindung zuruckkehrt, handelt es sich um Resociation. Die Jodreaction des Glykogenes muss also vom Standpunkt der Dissociation lockerer Verbindungen erklärt werden.

Dies macht dann auch die weitere Thatsache verständlich, dass beim Erhitzen eine Jodglykogenlösung s tärker als die Controlprobe. gefärbt bleibt, wenn ein gewisser U eberschuss von Jod vorhanden ist. Daraus folgt, dass bei $100^{\circ} \mathrm{C}$. die Resociation der Jodglykogenverbindung nicht vollständig aufgehoben ist. Die grössere Zahl freier Jodmolecüle sehafft trotz der dureh die hohe Temperatur bedingten verstärkten Dissociationsgeschwindiykeit die Möglichkeit, dass doch eine gewisse Zahl von Glykogenmolecülen sich in jedem Moment mit Jod sättigen kann.

Die mitgetheilten Thatsachen sind mit chemisch reinem Glykogen aus den Muskeln des Pferdes und aus der Leber des Hundes und Kaninchens gewonnen worden. - Ich verfehle nicht, hervorzuheben, dass ich auch zwei von Madame Gatin-Gružewska dargestellte und mir zur Verfügung überlassene Präparate desselben Glykogenes aus Hundeleber benutzen konnte, deren chemische Reinbeit von dieser Dame durch den Beweis der Freiheit von Stickstoff und Asche und viele andere Charakterisirungen bewiesen worden war, worüber sie bereits theilweise in ihrer Abhandlung ${ }^{1}$ ) ${ }_{n}$ über die Praecipitationserscheinungen des reinen Glykogenes" berichtet hat.

Es leuchtet ein, dass die mitgetheilten Thatsachen ermöglichen, die Jodmenge annähernd $z \mathfrak{u}$ bestimmen, welche durch eine gegebene Glykogenmenge in maximo gebunden zu werden vermag, sowie umgekehrt aus der zur Sättigung nöthigen Jodmenge die unbekannte Glykogenmenge zu berechnen.

Auch bleibt für den Analytiker zu beachten, dass beim Erhitzen einer Jodglykogenlösung die Reaction der "Entfärbung" nur dann gut gelingt, wenn kein erheblicher Jodüberschuss angewandt worden ist.

1) Madame Gatin-Grǔ̌ewska, dieses Archiv Bd. 100 s. 684.1903. 


\section{$\$$ 4. Die Jodglykogenreaction der Extracte der foetalen Leber.}

Wenden wir uns jetzt zur Anwendung der Jodreaction auf die aus der foetalen Leber erhaltenen neutral reagirenden Extracte. Höchst sonderbare Abweichungen treten auf.

Man fúllt eine mit Glashahn versehene Bürette mit einer JodJodkaliumlösung, die so concentrirt ist, dass ein kleiner Tropfen 5 cem Wasser kräftig gelb färbt. - Die Lösung enthielt $3,215 \%$ freies Jod. -

Von 2 Reagensgläsern gleichen Calibers füllt man das eine mit $5 \mathrm{ccm}$ des auf Glykogen zu prüfenden Leberauszugs, das andere mit $5 \mathrm{ccm}$ destillirten Wassers.

Ich beschreibe zuerst eine Reihe von Thatsachen, die man nicht erwartet, in der Reihenfolge, wie sie dem Leser das Verständniss erleichtern. Ich beobachtete, dass ein Tropfen Jodlösung in einem Leberauszug starke Braunfärbung erzeugte, welche beim rubigen Stehen des Reagensglases im Laufe einiger Stunden im Kalten von selbst spurlos verschwand. Niemals wird dies bei einer reinen Glykogenlösung beobachtet. War diese durch Erhitzen entfärbt worden, so stellt sich beim ruhigen Stehen im Kalten die Farbe vollkommen von selbst wieder her.

Die Schnelligkeit, mit welcher beim Stehen im Kalten die Färbung aus einer glykogenhaltigen Leberlösung verschwindet, ist sehr verschieden. Zuweilen erscheint nach Zusatz eines Tropfens der Jodlösung die braune Reaction, um beim Schütteln der Flüssigkeit sofort wieder zu verschwinden.

Es kommt sogar vor, dass trotz der Gegenwart von Glykogen ein 'Tropfen Jodlösung gar keine Farbenveränderung der Lösung hervorbringt.

Diese Thatsachen beweisen, dass in den der Untersuchung unterworfenen Extracten der foetalen Leber ein Stoff vorhanden ist, welcher das Jod fest chemisch bindet.

Es ist deshalb nicht auffallend, dass die Jodglykogenreaction aus solchen Extracten beim Erhitzen sehr schnell vollkommen bis zur Farblosigkeit verschwindet. Es ist ferner verständlich, dass beim Abkühlen der durch die Erhitzung entfärbten Lösung die Farbe nicht wiederkehrt. Setzt man aber einen zweiten Tropfen starker Jodlösung hinzu, so verschwindet die hierdurch wieder erzeugte tiefrothe Farbe beim Erhitzen abermals vollständig und kehrt beim 
Abkühlen nicht zurück. Das lässt sich oft 6 bis 10 Mal wiederholen zum Beweise, dass der das Jod fest bindende Körper sehr beträchtliche Jodmengen verschluckt, ehe er gesättigt ist. Je mebr man sich durch immer erneuten Jodzusatz der Sättigung nähert, um so langsumer findet die Bindung statt. Denn man muss immer längere Zeit bis zu vollkommener Entfärbung erwärmen. Die experimentelle Ermittelung des Sättigungspunktes bietet deshalb einige Schwierigkeit.

Hat man endlich vollkommene Sättigung der das Jod festbindenden Substanz erzielt, so verhält sich die Leberlösung dem Jod gegenüber wie die Lösungen chemisch reinen Glykogenes. Ein Tropfen Jodlösung erzeugt die entsprechende Färbung, welche durch Erhitzen bis zu dem Farbenton abblasst, den die Controlprobe darbietet. Es tritt also beim Kochen der Jodglykogenlösung keine vollkommene Entfärbung mehr ein, und stets kehrt beim Abkühlen die ursprüngliche kräftige Röthung wieder zurück. -

Es erscheint auf den ersten Blick auffallend, dass diese lockere Jodglykogenverbindung zu Stande kommen kann, wenn neben dem Glykogen ein das Jod fest bindender Stoff vorhanden ist.

Ist die Menge des fest bindenden Stoffes gross neben einer kleinen Glykogenmenge, beobachtet man thatsächlich wie bereits erwähnt, die sofortige Entfärbung der zugesetzten Jodlösung. -

Ist aber das Glykogen in verhältrissmässig grösserer Menge vorhanden, so tritt die rothbraune Jodreaction ein, gerade so wie ein Tropfen Silberlösung ein Lösungsgemenge von Chromat und Chlorid roth macht, obwohl auch hier die Entfärbung sich dann allmählich vollzieht, weil die stärkeren Affinitäten (des Chlors) auf Kosten der schwächeren (der Chromsäure) sich sättigen. Vielleicht handelt es sich auch bei der Störung der Jodglykogenreaction um mehr als einen einzigen Atomencomplex, der das Jod fest bindet, so dass noch secundäre Reactionen in Betracht kommen.

\section{$\S 5$. Ueber die Jodreaction der Extracte der Organe erwachsener Thiere.}

Es ist nothwendig, hervorzuheben, dass die das Jod fest bindende Substanz nicht etwa nur von der foetalen Leber geliefert wird.

Sie lässt sich aus den Muskeln in sehr reicher Menge erhalten. Aus $100 \mathrm{~g}$ Pferdefleisch stellte ich mir Glykogen dar mit Hülfe meiner im Bd. 93 dieses Archives S. 163 genauer beschriebenen Methode. 
Der nach der Neutralisation wie oben bereits erwähnt filtrirte, schön opalisirende Auszug gab mit Sa l z äu re und Kaliumquecksil berjodid keine Fällung. Zur Verfügung waren ungefähr $50 \mathrm{cem}$ Lösung, in denen das Glykogen aus $100 \mathrm{~g}$ Pferdefleisch enthalten sein musste. Es war also eine starke Lösung.

Ein Tropfen einer sehr concentrirten Jod-Jodkaliumlösung zugefügt $\mathrm{zu} 5 \mathrm{cmm}$ dieses in einem gewöhnlichen Reagensglas befindlichen Muskelauszuges erzeugte dichte, undurchsichtige Röthung. Beim Erhitzen verschwand die rothe Farbe schnell vollständig und kehrte beim Abkühlen nicht zurück. Ein zweiter, dritter, vierter, fünfter, sechster Tropfen verhielten sich ebenso. Nach dem sechsten Tropfen erschien beim Abkühlen der nach dem Erhitzen nur schwach gelblich gefärbten Lösung eine geringe Röthung wieder. Der siebente Tropfen machte die Lösung abermals undurchsichtig. Die rothe Farbe verschwand nach dem Kochen nicht vollständig, kehrte aber fast vollständig beim Abkühlen wieder zurück. Das liess sich öfter wiederholen.

Es war nun die Frage, ob sich die das Jod bindende Substanz nicht vom Glykogen trennen lasse.

Ich hatte wenigstens in einem Falle gefunden, dass bei der nach Brücke-Külz ausgeführten Glykogenanalyse ein das Jod fest bindender Stoff in dem alkoholischen sauren Filtrat enthalten ist, aus welchem das Glykogen durch Filtration entfernt worden war. Ich wusste ausserdem, dass die durch das B ü ck e'sche Reagens bei der Glykogenanalyse nach Brücke-Külz gefällten Quecksilbereiweissverbindungen zum Theil leicht in Weingeist löslich sind. Ich suchte also mein Glykogen nach $\mathrm{K} \ddot{\mathrm{l}} \mathrm{z}$ zu reinigen, indem ich es mit Salzsäure und Kaliumquecksilberjodid versetzte, mit 2 Vol. Alkohol fällte und nach 12 Stunden abfiltrirte. Ich hatte die Hoffnung, dass der das Jod fest bindende Stoff ein Eiweissstoff sei, welcher mit Quecksilber eine in Alkohol lösliche Verbindung eingehen werde. Das gefällte Glykogen wurde zuerst wiederholt mit Alkohol von $66 \%$ Tr. gewaschen, wodurch die röthliche Farbe desselben nicht ganz beseitigt werden konnte. Ich wusch dann mit absolutem Alkohol, der zwar das Jod besser entzog, ohne aber vollkommen weisse Farbe zu erzielen. Ich löste deshalb das Glykogen in Wasser, fällte es nochmals mit Alkobol und erhielt so ein schneeweisses Präparat. Ich löste dasselbe in Wasser, neutralisirte bis $\mathrm{zu}$ sehr schwach alkalischen Reaction, verjagte den Alkohol auf dem Wasserbad und erhielt schliesslich ungefähr $40 \mathrm{cem}$ Lösung. 
Als ich mit diesem nach $\mathrm{Külz}$ gereinigten Glykogen nun die oben beschriebenen Versuche wiederholte, zeigte es sich, dass der das Jod fest bindende Körper noch ausgezeichnet kräftig vorhanden war. Denn die durch Jod undurchsichtig gemachte Glykogenlösung wurde durch Erhitzen vollkommen farblos, und durch Abkühlen konnte die Farbe nicht wieder hervorgerufen werden. Viele Male habe ich das an derselben Probe nach erneutem Jodzusatz wiederholen können, bis der Punkt eintrat, wo der das Jod fest bindende Körper gesättigt war. Dann tritt beim Erhitzen der rothbraunen Glykogenlösung nicht mehr vollständige Entfärbung ein, und beim Abkühlen stellt sich das Roth wieder her. Trotz der Gegenwart des Kaliumquecksilberjodids und Salzsäure war also doch ein sehr grosser Theil des jodbindenden Körpers mit dem Glykogen durch Alkohol gefällt worden.

Da ich noch einen Rest dieser aus Pferdefleisch dargestellten und nicht ausreichend gereinigten Glykogenlösung hatte, beschloss ich, eine Reinigung mit Hülfe der Reaction von P fl ü ger - N erk in g ${ }^{1}$ ) $\mathrm{zu}$ versuchen. Ieh füllte auf $\mathrm{zu} 100 \mathrm{ccm}$, die ich auf $3 \% \mathrm{KOH}+$ $10 \% \mathrm{JK}$ gebracht hatte, fällte mit $50 \mathrm{ccm}$ Alkohol von $96 \% \mathrm{Tr}$, filtrirte durch schwedisches Filter, wuseh zuerst mit einer Mischung von einem Vol wässriger Lösung von $3 \% \mathrm{KOH}$ und $10 \% \mathrm{JK}+$ 1/2 Vol Alkohol von $96 \% \mathrm{Tr}$, darauf mit Alkohol von $66 \%$ mehrmals, endlich mit Alkohol von 98,9\%, unter dem ich das Glykogen einige Zeit stehen liess, indem ich den Abfluss des Filtrates hinderte.

Nach Abfluss des Alkohols löste ich mit Wasser, verjagte den Alkohol auf dem Wasserbad, neutralisirte die abgekühlte Lösung mit Essigsäure. Jetzt war der das Jod fest bindende Körper verschwunden. Jetzt konnte durch Erhitzen der rothen Jodglykogenlösung kein vollkommenes Verschwinden durch selbst langes und wiederholtes Kochen mehr hervorgerufen werden, d. h. die Jorglykogenreaction verhielt sich in jeder Beziehung so, wie ich es für reine Glykogenlösungen beschrieben habe.

Es ist also hiermit ein Mittel gefunden, welches die Störung der Glykogenreaction durch den das Jod festbindenden Körper beseitigt. Man wird jetzt gewiss in vielen Fällen Glykogen an Orten nachweisen können, wo dies früher nicht gelingen konnte. Leider habe ich diese wichtige Thatsache erst entrleckt, nachdem ich die Unter-

1) E. Pflüger und J. Nerking, dieses Archiv Bd. 76 S. 581.1899. 
Fortges. Untersuchung über den Glykogengehalt der foetalen Leber ctc. 315

suchung über die Leber der jüngsten Foetalperiode bereits abgeschlossen hatte.

Beiläufig möge hier noch eine Reaction angegeben werden, durch die sich der das Jod fest bindende Stoff neben dem Glykogen verräth.

Versetzt man eine Lösung reinen Glykogenes mit Salzsäure und Kaliumquecksilberjodid, so bleibt sie für etwa eine Stunde und länger von annähernd unveränderter, schwach gelblicher Farbe. Allmählich wird das Gelb stärker ausgeprägt und geht in röthlichbraun über. Auch eine glykogenfreie solche Lösung zeigt allmählich diese oder eine ähnliche Aenderung. Offenbar zerlegt die Salzsäure das Jodid unter Freiwerden von Jodwasserstoff, der an der Luft sich allmählich unter Abscheidung von Jod oxydirt. Ist Glykogen in der Lösung, so tritt deshalb die Jodglykogenfarbe hervor. Die Bräunung beginnt im ruhig stehenden Reagensglas von der freien Oberfläche aus, wegen des Contacts mit dem atmosphärischen Sauerstoff. Wie mir schien, beschleunigt das Licht die Schnelligkeit der Bräunung.

Hat man aber eine Glykogenlösung, welche wie bei den Organextracten mit dem das Jod fest bindenden Körper verunreinigt ist, dann tritt bei Zusatz von Salzsäure und Kaliumquecksilberjodid die Jodglykogenreaction fast sofort ein und wächst schnell an Stärke. Es ist also ein Stoff da, welcher wie eine Oxydase wirkt. -

Nachdem der das Jod fest bindende Körper sowohl aus der foetalen Leber des Kalbes als aus den Muskeln des erwachsenen Pferdes erhalten worden war, lag die Folgerung nahe, dass dieser Stoff allgemein bei der Glykogenanalyse in Betracht komme.

Ich untersuchte deshalb noch die Leber des erwachsenen Kaninchens. $103 \mathrm{~g}$ Leberbrei wurden mit $103 \mathrm{ccm}$ Lunge von $60 \%$ KOH 3 Stunden im sieflenden Wasserbarl erhitzt und dann genau so verfahren, wie es für die foetale Leber und die Muskeln des Pferdes bereits beschrieben wurde. Da eine ganz ungeheure Menge von Glykogen gewonnen war, betrug die neutrale zur Untersuchung dienende Lösung $150 \mathrm{ccm}$.

5 ccm dieser Lösung, die also ungefähr dem Auszug aus nuı $3 \mathrm{~g}$ Leber entsprachen, setzten mich durch die ungeheure Stärk der jodbindenden Kraft in Erstaunen. Bis zu 38 Tropfen $=2 \mathrm{ccm}$ meiner concentrirten Jodlösung (sie war in der Bürette von $1 \mathrm{~cm}$ lichtem Durchmesser absolut undurchsichtig) wurden von diesen 5 cem Glykogenlösung vollständig entfärbt. In $1 \mathrm{ccm}$ der Jodlösung 
waren $0,03215 \mathrm{~g}$ Jod, also $0,06430 \mathrm{~g}$ Jod genügten erst zur Sättigung. Erst nach der Sättigung traten dann die Erscheinungen hervor, welches reine Glykogenlösungen darbieten.

Ich versuchte nun, ob es möglich sei, auch hier mit Hülfe der Kali-Jodkaliummethode das Glykogen so zu reinigen, dass die Lösung desselben Jod nicht mehr dauernd entfärbt. Dies gelang auch ausgezeichnet.

Die Reinigung nach $\mathrm{Br}$ ü cke-Kül z führte ich diesmal mit einer kleinen Abänderung aus. Die mit Salzsäure und Kaliumquecksilberjodid versetzte Glykogenlösung gab keine Fällung; aber die Opalescenz der Lösung hatte zugenommen, weshalb ich mehrmals durch ein schwedisches Filter filtrirte und aus dem Filtrat dann in bekannter Weise das Glykogen isolirte.

Dieses nach $\mathrm{Brücke-Külz}$ gereinigte Glykogen enthielt nur noch Spuren des Jod fest bindenden Körpers. Dieser war also dadurch beseitigt worden, dass die durch das Brücke'sche Reagens erzeugte Trübung durch Filtration ziemlich beseitigt werden kounte. Auf dem Filter liess sich eben ein Hauch eines bräunlichen Ueberzugs bemerken.

Verfehlen will ich nicht, hervorzuheben, dass ich bei der Reinigung des Glykogenes nach den beiden Methoden niemals versäumte, durch Alkohol von $66 \%$ Tr. aus dem gefällten Glykogen das Jodkalium beziehungsweise das Kaliumquecksilberjodid und Salzsäure auszuwaschen.

Mit Rücksicht auf die Entfärbung, welche der das Jor fest bindende Körper in Glykogenlösungen hervorbringt, muss noch hervorgehoben werden, dass diese Entfärbung im strengsten Sinne des Wortes sich um so weniger absolut vollständig vollzieht, je näher man nach Zusatz grösserer Jodmengen dem Sättigungspunkte kommt. Die Lösung behält dann selbst bei wiederholtem Kochen einen schwachen Stich in's Röthliche, der besonders gegen einen weissen Hintergrund betrachtet einer schwachen Glykogenjodreaction ähnelt. Dass es sich aber nicht um diese handelt, erkennt man daran, dass die Abkühlung der Lösung nach dem Erhitzen keine Spur einer Wiederkehr der stärkeren Röthung bedingt, und dass erneutes Erhitzen keine Fntfärbung erzeugt. Der Zusatz einer Spur Jod bewirkt augenblickiich die dunkle Röthung, welche beim Kochen wieder nicht ganz vollständig, sondern nur bis zu dem genannten schwach gelbröthlichen 
Fortges. Untersuchung über den Glykogengehalt der foetalen Leber etc. 317

Ton entfärbt wird. Beim Ablühlen erscheint die stark rothe Tarbe anf's Neue.

Der das Jod fest bindende Körper hat also auch eine röthliche Farbe, weshalb Organextracte, welche kein Glykogen oder nur Spuren desselben enthalten, leicht za Täuschungen führen. Unter steter Benutzung der Controlprobe muss festgestellt werden, dass beim Erhitzen die röthliche Farbe sich nicht nur umändert, sondern auch, dass sie beim Abkühlen wiederkehrt. Wünschenswerth bleibt dann immer die Reinigung des Glykogenes mit Hülfe der JodkaliumKalimethode oder doch die Sättigung des das Jod fest bindenden Körpers vor Anstellung der definitiven Jodglykogenreaction.

Es ist von Interesse noch nach der chemischen Natur des so stark das Jod fest bindenden Körpers zu fragen. Was bis jetzt vorliegt, genügt zu einem sicheren Urtheil keineswegs. Die mitgetheilten Reactionen deuten aber auf einen Eiweissstoff. Denn die jodbindende Substanz wird aus wässriger Lösung durch Salzsäure und Kaliumquecksilberjodid gefällt; und diese Fällung ist unlöslich oder schwer löslich in Alkohol. Ebenso sind die Verunreinigungen des Glykogenes, welches auch immer die Methode der Darstellung war, stets stickstoffhaltig. Nicht im Einklang mit der Annahme, dass es sich um einen Eiweissstoff handelt, ist die Thatsache, dass die vielen von verschiedenen Forschern ausgeführten Elementaranalysen des (unreinen) Glykogenes immer viel zu niedrige Werthe für den Kohlenstoff ergeben haben. Eine Verunreinigung des Glykogenes mit Eiweiss müsste den procentigen Kohlenstoffgehalt steigern. Nun könnte ja allerdings die Verunreinigung ein Gemenge sein. -

Auffallend ist auch die geringe Menge des Jod fest bindenden Stoffes. Denn das Glykogen aus der Kaninchenleber, welches die ungeheuer starke Jod fest bindende Wirkung zeigte, verlor sie nach Ausfällung durch das Brücke'sehe Reagens fast vollständig. Auf dem Filter, das den wirksamen Stoff zurückgehalten hatte, befand sich nur ein röthlicher Hauch.

Durch diese Untersuchung ist nun eine Möglichkeit eröffnet, mit Hoffnung auf Erfolg eine quantitative Anaiyse des Glykogenes durch Colorimetrie auszuarbeiten. Es scheint mir aber, dass immer für angenäherte Bestimmungen meine Kalimethode der quantitativen Analyse genauer ist und schneller zum Ziele führt, wenn man den durch Inversion des Glykogenes erhaltenen Zucker titrirt. Ich hoffe durch die von mir neu gefundenen und erklärten Störungen der 
Glykogenreaction noch schärfer meine Einwände gegen die von Jensen ${ }^{1}$ ) empfohlene Colorimetrie des Glykogenes erweitert und befestigt zu haben.

\section{$\$$ 6. Ueber den Nachweis des Glykogenes durch Inversion.}

Obwohl nun die Ausführung der Jodglykogenreaction in der von mir gemeldeten Form genügt, um das Glykogen mit Sicherheit nachzuweisen, bleibt es bei einer wichtigen Frage wünschenswerth, das Ergebniss durch Inversion des Glykogenes zu bestätigen.

Mein Verfahren war folgendes:

Nachdem, wie oben beschrieben, das alkalische, glykogenhaltige Filtrat zur Verjagung des Alkohols auf ein kleines Volum gebracht ist, wird dasselbe mit Salzsäure neutralisirt und mit den ausgeschiedenen Flocken in ein $50 \mathrm{ccm}-\mathrm{Kölbchen} \mathrm{gegossen,} \mathrm{auf} 2,2 \% \mathrm{ClH}$ gebracht und drei Stunden im Wasserbad erhitzt. Dann filtrirt man durch ein kleines Filter, misst das Volum des Filtrates. Es seien $30 \mathrm{ccm}$, welche in ein Becherglas gegossen und genau mit Lauge von $60 \% \mathrm{KOH}$ neutralisirt werden. In dasselbe Becherglas giesst man $30 \mathrm{ccm}$ der Allihn'schen Kupferlösung. - Zur Controle bringt man in ein gleich grosses zweites Becherglas $30 \mathrm{ccm}$ Wasser $+30 \mathrm{ccm}$ Allihn'sche Kupferlösung. Dann erhitzt man beide mit Uhrgläsern bedeckte Bechergläser gleichzeitig 30 Minuten im Wasserbade, stellt auf weisses Papier, wartet eine Stunde und überzeugt sich dann, dass nur die zuckerhaltige Lösung eine grössere Abscheidung von Kupferoxydul aufzeigt.

Handelt es sich um erhebliche Zuckermengen oder um quantitative Analyse, verfährt man nach den von mir angegebenen Vorschriften $\left.{ }^{2}\right)$.

\section{§ 7. Ergebnisse.}

Als wesentliches Ergebniss dieser Untersuchung ist zu beachten:

1. Es liegt keine Berechtigung $\mathfrak{z}$ der Annahme vor, dass die foetale Leber in den früheșten Entwicklungsperioden kein Glykogen enthalte.

Ob die Leber während der ersten Foetalentwicklung grundsätzlich ärmer an Glykogen sei als in der Zeit nach der Geburt, kann erst durch zukünftige Untersuchungen entschieden werden.

1) Paul Jensen, Zeitschr. f. physiol. Chemie Bd. 35 S. 525.

2) E. Pflüger, dieses Archiv Bd. 93 S. 163. 1902. 
Fortges. Untersuchung über den Glykogengehalt der foetalen Leber etc. 319

2. Die rothe Jodglykogenverbindung ist eine lockere, für welche die Gesetze der in Dissociation verkehrenden chemischen Verbindungen maassgebend sind.

3. Die foetale Leber sowie die Leber und Muskeln erwachsener Thiere liefern nach der Lösung in Kalilauge das Jod fest bindende Stoffe, welche die Glykogenreaction oft erheblich stören, aber nach den im Text beschriebenen Methoden beseitigt werden können. 